\title{
Merleau-Ponty, Foucault et l'autonomie de la conscience
}

\author{
Par CLAUDIO CORMICK \\ CONICET - Université de Paris 8 Vincennes-Saint-Denis - Universidad de Buenos \\ Aires
}

Résumé Nous essayerons dans ce travail d'analyser le problème du caractère conditionné ou autonome du sujet de la connaissance, en prenant comme point de départ la mise en rapport proposée par Philippe Sabot, dans un article récent, entre les thèses de Michel Foucault dans Les mots et les choses et celles de Maurice Merleau-Ponty dans son cours Les sciences de l'homme et la phénoménologie. D'abord (section 1) nous reconstruirons brièvement la lecture de Sabot, selon laquelle les deux philosophes auraient accordé sur un même «diagnostic» initial sur la dualité empirico-transcendantale de la condition humaine, malgré les différences de leurs solutions au dilemme. Ensuite (section 2), nous rappellerons que la «dualité » des vues sur l'homme consiste, pour le phénoménologue, en la tension entre une approche à la conscience comme « autonome » ou comme «conditionnée » par son milieu, et essaierons une reconstruction de l'argument selon lequel une telle dualité ne peut pas être simplement dépassée par le biais d'une acceptation unilatérale de la deuxième thèse. Le pas suivant (section 3 ) sera d'analyser si c'est bien d'une telle contradiction qu'il s'agit lorsque Foucault se réfère au rapport entre 1' « empirique » et le «transcendantal » — une lecture qui a été défendue non seulement par Sabot mais aussi par d'autres lecteurs de Foucault comme Jürgen Habermas. Au contraire, nous allons argumenter que - précisément parce que Foucault parle d'une « répétition » de l'empirique au niveau du transcendantal, d'une «valeur » transcendantale donnée aux contenus empiriques - il n'y a chez l' « archéologue » aucune adhésion à la thèse que la dualité entre ces deux vues sur la conscience concernerait la dichotomie autonomie-conditionnement. Par ailleurs (section 4), l'archéologue n'a pas même besoin d'aborder un « dilemme » comme celui qui existe pour Merleau-Ponty, parce que, comme il le déclare dans plusieurs textes, le 
caractère historiquement et socialement conditionné de la conscience n'empêche pas la capacité de celle-ci à distinguer le vrai du faux.

\section{Le point de départ : sur une récente interprétation du rapport entre Foucault et Merleau-Ponty}

Dans un article de l'année 2013, Philippe Sabot a tenté un rapprochement entre la pensée de Foucault, telle qu'elle apparait dans le chapitre 9 des Mots et les choses, et celle de Merleau-Ponty dans le point de départ de son cours sur Les sciences de l'homme et la philosophie: dans les deux cas, selon Sabot, on a affaire à des problématisations d'un certaine « dualité » qui aurait affecté quelques analyses classiques sur l'être humain, vu alternativement comme un sujet et comme un objet pour la connaissance. Nous essayerons de montrer dans ce travail qu'une telle assimilation des perspectives de ces deux philosophes obscurcit une différence fondamentale: c'est selon MerleauPonty, mais pas selon Foucault, que le sujet de la connaissance peut l'être seulement à la condition qu'il soit autonome, et donc ne peut pas être considéré comme un objet conditionné par son milieu. Foucault, par contre, ne semble pas de tout accepter cette prémisse merleau-pontienne, et donc son approche à la dualité de l'homme est tout à fait différente.

Voyons d'abord l'interprétation proposée par Sabot. L'auteur de Lire Les Mots et les Choses de Michel Foucault prend pour point de départ, dans cette approche plus récente, les très connues références de Foucault à l' « homme » de l'épistémè moderne comme un « doublet empirico-transcendantal » et déclare que

Foucault déploie en grande partie sa critique des sciences humaines à partir du diagnostic formulé par Merleau-Ponty dans les premières lignes du cours intitulé «Les sciences de l'homme et la phénoménologie » [...]. [L]a crise qui affecte les sciences de l'homme tient, selon Merleau-Ponty, à [la] tension qu'elles manifestent entre le point de vue de l'objectivité empirique et le point de vue de la subjectivité transcendantale ${ }^{1}$.

${ }^{1} \mathrm{Ph}$. Sabot, «Foucault et Merleau-Ponty : un dialogue impossible ?», Les Études philosophiques, 106/3 (2013), p. 323. 
En effet, même si la «solution phénoménologique » consistant à réunir les deux aspects sous l' " ambigu» mode d'être de l'homme n'était pas acceptable pour Foucault ${ }^{1}$, il n'en reste pas moins que, ajoute Sabot,

c'est bien le diagnostic merleau-pontien de la crise des sciences de l'homme qu'il installe au cœur du dispositif anthropologique de l'épistémè moderne. Foucault montre en effet que l'homme qui se trouve au centre de la pensée moderne apparaît comme « un étrange doublet empirico-transcendantal » qui se présente à la fois comme l'objet et le sujet de son propre savoir ${ }^{2}$.

Sabot a sans doute raison de dire qu'il existe une similitude entre les approches de Merleau-Ponty et de Foucault. Cependant, les deux auteurs s'accordent-ils vraiment sur un même «diagnostic », ne divergeant que par les solutions qu'ils proposent ? Voyons, dans leurs contextes, ce qu'ils ont à dire sur la condition de l'homme.

\section{Merleau-Ponty : sur deux « vues» incompatibles sur l'homme}

Le point de départ de Merleau-Ponty dans son cours sur Les sciences de l'homme et la phénoménologie reprend, dans une certaine mesure, sa référence aux «deux vues classiques » sur l'homme déjà présentée dans son article «La querelle de l'existentialisme», où le phénoménologue rejetait aussi, avec la vision cartésienne de l'homme comme conscience inconditionnée, celle qui explique cette conscience comme un objet déterminé par des conditionnements extérieurs ${ }^{3}$. Mais les raisons de cette double critique apparaissent plus clairement dans le contexte du cours de la Sorbonne, où Merleau-Ponty constate le dépassement de la réflexion philosophique par les connaissances "positives" sur l'homme. D'une part, le phénoménologue accepte en principe le rôle d'une connaissance «extérieure » sur l'homme, laquelle ne requiert pas que le sujet connaissant coïncide avec ce qui est connu, et peut donc être obtenue « en troisième personne ». Si cette connaissance de l'homme comme un objet conditionné pouvait être satisfaisante, on aurait donc réduit celui-ci à cette seule dimension. Mais d'autre part - et c'est ici que le problème de la dualité se révèle difficile à surmonter -, le point de Merleau-Ponty est que ce qui connaît « de dehors" les autres sujets est luimême un sujet humain, et sa conscience a besoin d'avoir l' "autonomie "

${ }^{1} C f$. ibid., p. 324.

${ }^{2}$ Ibid.

${ }^{3}$ M. Merleau-Ponty, Sens et non-sens, Paris, Nagel, 1948, p. 142. 
nécessaire pour accéder à ses propres évidences, sans lesquelles il n'y aurait pas de justification épistémique et donc pas de connaissance, même « extérieure ». La connaissance en troisième personne requiert qu'au moins une conscience soit au moins partiellement autonome : celle, «objective», du savant qui doit savoir pourquoi il dit ce qu'il dit sur l'opacité de la conscience de tous les autres. Voyons ce que Merleau-Ponty dit au début même du cours :

Les recherches psychologiques, sociologiques, historiques, à mesure qu'elles se développaient, tendaient à nous présenter toute pensée, toute opinion et [...] toute philosophie, comme le résultat de l'action combinée des conditions psychologiques, sociales, historiques extérieures. [...] Or, en le faisant, elles en venaient à déraciner leurs propres fondements. Si en effet, les pensées et les principes directeurs de l'esprit, ne sont à chaque moment que le résultat des causes extérieures qui agissent sur lui, les raisons pour lesquelles j'affirme quelque chose ne sont pas en réalité les vraies causes de mon affirmation. Elle a moins des raisons que des causes, causes qu'il s'agit de déterminer de l'extérieur. Si bien que les postulats du psychologue, du sociologue ou de l'historien, sont frappés de doute par le résultat même de leurs recherches ${ }^{1}$.

Et ceci parce que l'on devrait appliquer aux savoirs scientifiques les mêmes critères qui, selon ceux-ci, disqualifient la philosophie ${ }^{2}$. Dans un autre passage du même cours, et cette fois spécifiquement sur le psychologisme, Merleau-Ponty ajoute que si celui-ci

nous dit que le philosophe et sa pensée ne sont que des marionnettes, des mécanismes psychologiques ou de l'histoire extérieure, on peut toujours lui

\footnotetext{
${ }^{1}$ M. Merleau-Ponty, Les sciences de l'homme et la phénoménologie, Paris, Centre de Documentation Universitaire, 1963, p. 1.

${ }^{2}$ Ibid., p. 2 : «Quant à la philosophie, elle perdait, dans ces conditions, toute espèce de justification. Comment prétendre encore comme philosophe qu'on détient des vérités, et des vérités éternelles, alors qu'il est manifeste que les différentes philosophies replacées dans le cadre psychologique, social et historique auquel elles appartiennent, ne sont rien d'autre que des expressions de ces causes extérieures ? Pour que le philosophe puisse professer la philosophie, pour qu'il puisse faire une distinction entre le vrai et le faux, il faut que ses énoncés expriment, non pas quelques conditions naturelles ou historiques extérieures à lui, mais un contact direct et intérieur de l'esprit avec l'esprit, une vérité "intrinsèque" qui paraît impossible, alors que le développement des recherches dans le domaine des sciences de l'homme montre à chaque instant qu'au contraire l'esprit est extérieurement conditionné ».
} 
répondre qu'il en va de même pour lui, et discréditer ainsi sa propre critique. Le psychologisme, du moins s'il est conséquent, devient un scepticisme radical : scepticisme à l'égard de lui-même ${ }^{1}$.

Quelque chose de semblable arriverait, selon Merleau-Ponty, au sociologisme : il est

exposé aux mêmes conséquences. À force de montrer que toute notre pensée est l'expression d'une situation sociale, dont les limitations font aussi que cette pensée n'est pas vraie, on s'expose à prouver trop, à accréditer l'idée que le sociologisme, lui aussi, n'a pas son vrai sens en lui-même [...]. C'est contre ces dangers $[\ldots]$ que $[\ldots]$ la tâche du philosophe [est de] restaurer la certitude, restaurer la distinction du vrai et du faux ${ }^{2}$.

En abrégé, l'argument merleau-pontien a une forme tu quoque: si l'on accepte que la non-autonomie de la conscience, sa dépendance, valent comme un argument contre les prétentions de la philosophie à être « connaissance ", on doit accepter aussi qu'elles valent comme un argument contre le psychologisme et le sociologisme eux-mêmes; ou bien la dépendance à l'égard de son milieu ne pose problème pour aucune pensée, ou bien elle le pose pour toutes ; tertium non datur ${ }^{3}$. Schématiquement,

(1) Le psychologiste (sociologiste, historiciste, etc.) caractérise la conscience humaine comme conditionné au sens spécifique que nos prises de position ont des causes qui nous échappent et donc que nous ne contrôlons pas; ce ne seraient pas nos raisons conscientes qui soustendent ces prises de position.

Mais cela n'a de conséquences importantes que si l'on ajoute une deuxième prémisse :

\footnotetext{
${ }^{1}$ Ibid., p. 7. Nous soulignons.

2 Ibid. Nous soulignons.

${ }^{3}$ Avec des différences, l'argument de Merleau-Ponty ici rappelle les avertissements de Max Weber sur la possibilité pour les marxistes de s'exempter eux-mêmes ad hoc de ce qu'ils dénonçaient chez leurs rivaux : «L'interprétation matérialiste de l'histoire n'est pas $[\ldots]$ un fiacre dans lequel on peut monter à son gré et qui s'arrêterait devant les promoteurs de la révolution » (Le savant et le politique, Paris, Union Générale d'Éditions, 1963, p. 180).
} 
(2) Une conscience ainsi conditionnée n'est pas capable de distinguer le vrai du faux.

De (1) et (2) s'ensuit, selon Merleau-Ponty, que

(3) le psychologiste met en cause nos prétentions de connaissance, il est donc un sceptique par rapport à elles.

Mais, d'un autre côté, ajoute Merleau-Ponty, le psychologiste ne peut pas légitimement s'exempter :

(4) Le psychologiste est lui-même un sujet humain (c'est-à-dire que, s'il est cohérent, il doit s'inclure lui-même sous la thèse (1)).

Donc, finit le phénoménologue,

(5) le psychologiste met en cause, en fait, ses propres prétentions à la connaissance ; il est un sceptique par rapport à lui-même.

La thèse (5), bien sûr, n'est pas présentée par Merleau-Ponty comme quelque chose à laquelle le psychologiste adhérerait; celui-ci n'apparaît pas dans l'argument comme quelqu'un qui tombe consciemment dans l'auto-réfutation, mais comme quelqu'un dont la position est objectivement autoréfutante ; évidemment, ce que le phénoménologue essaie de montrer, c'est que, pour être « consistent », on doit accepter (5) si l'on a déjà accepté (1) et (4). Mais cela arrive en raison du même principe (2) qui est censé être accepté par le psychologiste lorsqu'il accepte le mouvement de (1) à (3) : si l'opacité de nos croyances est un argument acceptable pour disqualifier la capacité $d u$ philosophe de distinguer le vrai du faux, on ne peut pas suspendre cette prémisse quand il s'agit du savant.

Nous voyons donc pourquoi il y a un problème avec la condition «double» de l'homme selon Merleau-Ponty: on ne peut pas dénier les découvertes de la science positive, mais l'homme ne peut pas être seulement le type d'entité conditionné que celle-ci nous présente lorsqu'elle parle de lui; donc, nous avons deux considérations incompatibles (l'homme comme conditionné, l'homme comme autonome) et ne pouvons, en principe, renoncer à aucune. Étant donné que notre intérêt ici est d'évaluer l'interprétation 
sur un prétendu « diagnostic » commun à Merleau-Ponty et à Foucault, nous ne nous référerons pas ici à la solution merleau-pontienne proposée dans la suite du cours. Retenons seulement que, selon Merleau-Ponty,

- Il y a une crise des sciences humaines parce que les connaissances sur l'homme en première et en troisième personne, telles qu'elles ont été développées jusqu'à certain moment, sont incompatibles, qu'elles se démentent l'une l'autre, dans la mesure où elles présentent la conscience comme autonome ou comme déterminée ;

- mais par ailleurs, on ne peut pas adopter seulement la vue sur l'homme qui fait de lui un objet déterminée par son milieu, parce que cette thèse se réfute elle-même.

Voyons maintenant si on trouve un problème semblable chez Foucault.

\section{Un retour sur le texte des Mots et les choses: qu'est-ce que la duplication empirico-transcendantale?}

a) La décomposition de l'épistémè classique et le problème de la synthèse

Pour arriver à comprendre la notion d'un « doublet » présentée dans le chapitre 9 des Mots et les choses, nous devons retourner au contexte beaucoup plus général constitué par l'analyse de l'épistémè en question comme celle où un nouveau type d'être, celui de l'homme, prend à sa charge la possibilité de la connaissance. Le problème de la connaissance n'avait pu apparaître lié au rôle d'une certaine subjectivité, selon Foucault, qu'à partir du XVIII siècle, parce que c'est seulement à ce moment (c'est-à-dire, à partir de la fin de ce que Foucault appelle l'Âge Classique) que la connaissance de la réalité cesse d'apparaître comme le résultat d'une mise en ordre des représentations des choses. En abrégé, ce qui se passe dans la transition entre l'Âge Classique et la Modernité, c'est que

ce qui valorise les objets du désir, ce ne sont pas seulement les autres objets que le désir peut se représenter, mais un élément irréductible à cette représentation, le travail ; ce qui permet de caractériser un être naturel, ce ne sont plus les éléments qu'on peut analyser sur les représentations qu'on se fait de lui et 
des autres, c'est un certain rapport intérieur à cet être et qu'on appelle son organisation; ce qui permet de définir une langue, ce n'est pas la manière dont elle représente les représentations, mais une certaine architecture interne, $[\ldots]$ son système flexionnel ${ }^{1}$.

La connaissance a certes, pour l'Âge Classique, un aspect « constructif » le besoin d'analyser ces représentations en identifiant des unités élémentaires, puis de reconstruire l'ordre continu du réel à partir des opérations de synthèse $^{2}$-, mais ces unités (par exemple les caractères visibles dans l'histoire naturelle de Linné) ne se situent pas à un autre « niveau » que celui de ce qui est donné à la représentation. Par contre, l'émergence des analyses scientifiques centrées sur de nouvelles entités telles que le travail, la vie et le langage met le principe de la synthèse, de la possibilité d'un ordre des richesses, du vivant et des langues, au-delà de ce que nous pouvons retrouver dans ceux-ci ${ }^{3}$.

D'abord, en ce qui concerne les richesses, l'analyse des représentations - désormais situées à un niveau «superficiel» - ne suffit pas à révéler les lois spécifiques expliquant l'échange des marchandises : depuis Ricardo, les marchandises ne sont pas considérées comme interchangeables parce qu'elles peuvent «se représenter les unes les autres » ${ }^{4}$, comme selon l'analyse classique des richesses, mais parce que leur valeur « tire son origine du travail $»^{5}$, c'est-à-dire d'une source qui est quelque chose de plus qu'une autre marchandise dans le tableau d'équivalences; le travail est plutôt le principe sous-jacent au tableau lui-même. En deuxième lieu, l'analyse des représentations - qui, dans l'histoire naturelle classique, impliquait l'identification dans les êtres vivants des unités perceptibles dont la combinatoire

\footnotetext{
${ }^{1}$ M. Foucault, Les mots et les choses, Paris, Gallimard, 1966, p. 250.

${ }^{2}$ Cf. ibid., p. $82-84$ et 320.

${ }^{3}$ Foucault résume l'avènement de la Modernité en rappelant que «Cuvier et ses contemporains avaient demandé a la vie de définir elle-même, et dans la profondeur de son être, les conditions de possibilité du vivant ; de la même façon, Ricardo avait demandé au travail les conditions de possibilité de l'échange, du profit et de la production; les premiers philologues avaient aussi cherché dans la profondeur historique des langues la possibilité du discours et de la grammaire. Du fait même, la représentation a cessé de valoir, pour les vivants, pour les besoins et pour les mots, comme leur lieu d'origine et le siège primitif de leur vérité ; par rapport à eux, elle n'est rien de plus, désormais, qu'un effet, leur répondant plus ou moins brouillé dans une conscience qui les saisit et les restitue » (ibid., p. 322-323; nous soulignons).

${ }^{4}$ Ibid., p. 266.

${ }^{5}$ Ibid.
} 
permettait de les classer ${ }^{1}$ - se révèle également insuffisante pour comprendre l'organisation des êtres vivants selon des fonctions nécessaires à la vie, fonctions qui ne s'identifient pas avec des traits observables spécifiques $^{2}$. Quant au langage, en troisième lieu, Foucault essaie de montrer que « le mot subit, à peu près à la même époque » que les changements concernant le statut du vivant et les richesses, « une transformation analogue » en vertu de laquelle sa fonction représentative devient secondaire par rapport aux «lois strictes » qui régissent chaque langue, et dont le lieu est plus profond que celui de la représentation ${ }^{3}$. Ainsi, dans ces trois domaines, « la représentation a perdu le pouvoir de fonder, à partir d'elle-même dans son déploiement propre [...], les liens qui peuvent unir ses divers éléments ${ }^{4}$. La « condition de ces liens », au lieu d'être trouvable au niveau des représentations elles-mêmes, "réside désormais à l'extérieur de la représentation, audelà de son immédiate visibilité, dans une sorte d'arrière-monde plus profond qu'elle-même $»^{5}$.

Le problème de ces liens, c'est-à-dire, le problème de la synthèse, est, selon Foucault, ce qui donne lieu à la philosophie transcendantale, laquelle « interroge les conditions d'un rapport entre les représentations du côté de ce qui les rend en général possibles », et ainsi découvre " un champ transcen-

\footnotetext{
${ }^{1}$ L'histoire naturelle classique, que Foucault distingue avec insistance de la biologie, n'est pour l'archéologue « rien d'autre que la nomination du visible» (ibid., p. 144), la «transcription» de «la visibilité de l'animal ou de la plante [...] dans le discours qui la recueille » (ibid., p. 147). C'est-à-dire, la tâche de cette science est avant tout celle d'une description de chaque espèce vivante, description organisée à travers la considération de quelques variables perceptibles rigoureusement limitées («forme des éléments, quantité de ces éléments, manière dont ils se distribuent dans l'espace les uns par rapport aux autres, grandeur relative de chacun »; ibid., p. 146) et à travers laquelle "chacun pourra reconnaître les individus qui y correspondent» (ibid.).

${ }^{2}$ Foucault prend ici l'exemple de Cuvier. Dans la biologie de celui-ci, «la diversité visible des structures n'émerge plus sur le fond d'un tableau de variables, mais sur le fond de grandes unités fonctionnelles susceptibles de se réaliser et d'accomplir leur but de manières diverses » (Les mots..., p. 276). On est donc passé «à l'évidente invisibilité de la fonction. Les branchies et les poumons», par exemple, "peu importe après tout s'ils ont en commun quelques variables de forme, de grandeur, de nombre : ils se ressemblent parce qu'ils sont deux variétés de cet organe inexistant, $[\ldots]$ absent de toute espèce descriptible [...] et qui sert à respirer en général » (ibid., p. 277, souligné dans le texte).

${ }^{3}$ Ibid., p. 293.

${ }^{4}$ Ibid., p. 251.

${ }^{5}$ Ibid., p. 252.
} 
dantal où le sujet [...], qui n'est jamais donné à l'expérience (puisqu'il n'est pas empirique), [...] détermine [...] toutes les conditions formelles de l'expérience en général $»^{1}$. La philosophie kantienne se distingue donc de la pensée classique en ce que celle-là cherche à expliquer les représentations par des conditions externes à la représentation elle-même, mais elle se distingue aussi des "métaphysiques de l'objet » en ce que c'est le sujet - et non pas le contenu représenté - qui joue ce rôle explicatif de synthèse ${ }^{2}$.

Cependant, Foucault trouve chez Kant non seulement l'introduction du thème transcendantal, mais aussi le germe de l'anthropologisme à travers lequel ce thème aurait été dégradé par les philosophies "modernes» postérieures à celle du père de l'idéalisme allemand, en raison de leurs efforts pour chercher dans des caractères empiriquement connus les conditions de toute connaissance. Il s'agit, en d'autres mots, de la constitution de l'homme en tant que « doublet empirico-transcendantal $»^{3}$ - le point où, selon Sabot, la réflexion de Foucault sur l'homme s'inspirerait de celle de Merleau-Ponty.

\section{b) L'analytique de la finitude et la «valeur » transcendantale des contenus empiriques}

La connaissance empirique, mais élevée à un statut «transcendantal», à laquelle se réfère Foucault peut être de deux types. D'un côté, il s'agit des analyses sur les conditions naturelles, anatomo-physiologiques, de la connaissance humaine: ceux qui s'associent au fait que nous connaissons le

\footnotetext{
${ }^{1}$ Ibid., p. 256.

${ }^{2}$ Ces métaphysiques, selon Foucault, interrogent aussi « les conditions d'un rapport entre les représentations », mais « du côté de l'être même qui s'y trouve représenté ». Ibid., p. 256.

3 « À partir de Kant, dit Foucault, se pose le problème des rapports entre le domaine de l'empiricité et le fondement transcendantal de la connaissance » (ibid., p. 260, nous soulignons), et c'est pourquoi une philosophie telle que la phénoménologie fonctionne dans le cadre de l'héritage kantien. En raison de l'intérêt de la phénoménologie à « lier [...] la subjectivité transcendantale à l'horizon implicite des contenus empiriques », Foucault avertit : «Il n'est sans doute pas possible de donner valeur transcendantale aux contenus empiriques ni de les déplacer du côté d'une subjectivité constituante, sans donner lieu, au moins silencieusement, à une anthropologie, c'està-dire à un mode de pensée où les limites de droit de la connaissance (et par conséquent de tout savoir empirique) sont en même temps les formes concrètes de l'existence telles qu'elles se donnent précisément dans ce même savoir empirique » (ibid., p. 261).
} 
monde à travers de nos sens, de notre système nerveux; tout cela aurait constitué selon Foucault une «quasi-esthétique transcendantale » ${ }^{1}$. D'un autre côté, on trouve les études qui se rapportent aux conditions historiques, socio-culturelles, de la connaissance, ou mieux, de l'illusion et de sa progressive disparition ${ }^{2}$. Ces deux entreprises théoriques ne s'appuient pas selon l'archéologue sur une "réflexion pure » comme la critique kantienne, mais sur certains «partages » dont on a besoin si une analyse de la nature et de l'histoire de la connaissance doit être possible : d'abord le partage entre les esquisses sensorielles du savoir et sa forme finale ; en deuxième lieu la division entre l'idéologie ou l'illusion et la connaissance au sens strict ${ }^{3}$. Mais le partage principal, selon Foucault, est plus général : il s'agit de la division même du discours en raison de laquelle il y aurait un discours vrai sur les objets, les choses, mais aussi un deuxième discours vrai (métathéorique, dans un sens) qui pourrait se référer à la nature et à l'histoire de notre connaissance des objets ${ }^{4}$. Un problème central pour l'épistémè moderne serait par conséquent celui du rapport entre ces deux types de discours. Il y a ici, encore une fois, deux possibilités. Ou bien c'est le premier type qui domine le deuxième, parce que le discours métathéorique trouve son modèle dans le discours sur les choses, et on a donc affaire à une solution positiviste, dans laquelle toute vérité est explicitement réduite au statut de l'empirique ; les analyses épistémologiques deviennent aussi empiriques. Ou bien, en revanche, c'est le niveau méta-discursif qui doit " esquisser » les formes de notre connaissance des objets, connaissance qui est donc future, non encore existante, et l'analyse devient, dit Foucault, eschatologique ${ }^{5}$. C'est dans le

\footnotetext{
${ }^{1}$ Ibid., p. 330.

${ }^{2}$ Ibid.

${ }^{3}$ Ibid.

${ }^{4}$ Ibid., p. 331. Comme dit Gary Gutting, il s'agit de « la distinction entre la vérité empirique impliquée dans notre connaissance biologique et historique des objets empiriques, et la vérité de notre discours philosophique sur cette connaissance » (G. Gutting, Foucault's Archaeology of Scientific Reason, Cambridge University Press, p. 201s.).

${ }^{5}$ Les mots..., p. 331. Nous ne pouvons ici qu'être en désaccord avec la lecture proposée par Leonard Lawlor et par Philippe Sabot, lesquels associent l'opposition positivisme-eschatologie avec celle qui existe entre la «quasi-esthétique » et la « quasi-dialectique ». En fait, toutes les deux, la première et la deuxième, sont des entreprises théoriques qui doivent résoudre, dans une direction ou une autre, le problème du rapport entre deux niveaux de connaissance, et pour lesquelles le « positivisme» et l' " eschatologie» sont des solutions alternatives. Cf. Ph. Sabot, Lire Les Mots et les Choses de Michel Foucault, Paris, Presses Universitaires de
} 
cadre de cette description d'un très fermé «espace de possibilités» de l'épistémè moderne que Foucault se réfère à l' "analyse du vécu " (une référence indirecte à Merleau-Ponty à travers le vocabulaire de la Phénoménologie de la perception) comme représentant d'une (impossible) troisième vue, ni positiviste ni eschatologique. Tandis que cette analyse voudrait faire du vécu «à la fois l'espace où tous les contenus empiriques sont donnés à l'expérience » et "aussi la forme originaire qui les rend en général possibles et désigne leur enracinement premier ${ }^{1}$, cette tentative, continue l'archéologue avec des mots un peu cryptiques, échoue: "La vraie contestation du positivisme et de l'eschatologie» ne se trouve pas, selon Foucault, «dans un retour au vécu (qui à vrai dire les confirme plutôt en les enracinant), mais si elle pouvait s'exercer, ce serait à partir d'une question qui sans doute semble aberrante [...]. Cette question consisterait à se demander si vraiment l'homme existe $\rangle^{2}$ — et c'est seulement cette question qui peut nous sortir de l'impasse, parce que « l'oscillation » entre un discours positiviste et un discours eschatologique est « inhérente à toute analyse qui fait valoir l'empirique au niveau du transcendantal $»^{3}$.

Mais, précisément parce qu'il s'agit ici de «faire valoir» les contenus empiriques au niveau transcendantal, il est clair que Foucault ne dit pas, en fait, que la double thématisation typiquement moderne du sujet d'un point de vue «empirique » et «transcendantal» est inconsistante, incohérente comme le pense Sabot et, avant lui, d'autres lecteurs importants de Foucault, y compris Jürgen Habermas ${ }^{4}$. Si des données empiriques peuvent être « em-

France, 2006, p. 130-131, et L. Lawlor, «Eschatology and positivism», Bulletin de la société américaine de philosophie de langue française, 14/1 (2004), p. 36.

${ }^{1}$ Ibid., p. 332.

${ }^{2}$ Ibid. Nous soulignons.

${ }^{3}$ Ibid., p. 331.

${ }^{4}$ Habermas analyse la notion de doublet empirico-transcendantal en disant que, «Depuis Kant, le Moi prend simultanément la position d'un sujet empirique se trouvant dans le monde comme un objet parmi d'autres objets, et celle d'un sujet transcendantal qui se place face au monde, lequel se constitue de lui-même comme totalité des objets de l'expérience possible. [...] La pensée ne peut naturellement pas s'accommoder de ces alternatives inconciliables. De Hegel à Merleau-Ponty, on a essayé de surmonter ce dilemme en fusionnant ces deux aspects en un, et de concevoir l'histoire concrète des formes a priori comme un processus d'autogénération de l'esprit ou de l'espèce » (J. Habermas, «Les sciences humaines démasquées par la critique de la raison : Foucault», dans Le débat, 1986, n 41, p. 90 ; nous soulignons). Beaucoup plus récemment, c'est la même idée d'une contradiction que Lee Braver trouve dans son analyse de Foucault lorsqu'il le lit de la manière suivante : 
ployées » pour déterminer des conditions de la connaissance - de la façon dont le fait par exemple la phénoménologie lorsque, comme Foucault à la fin du chapitre VII des Mots et les choses, elle donne «valeur transcendantale aux contenus empiriques $»^{1}-$, cela veut dire que la dynamique que Foucault trouve entre les dimensions transcendantale et empirique de l'homme n'est pas celle d'un démenti; il ne s'agit pas d'une tension où les découvertes positives, scientifiques, visent à montrer l'homme comme conditionné par son milieu tandis que les analyses transcendantales essaient de prouver, par contre, que l'homme doit être considéré comme une subjectivité autonome sans laquelle on ne pourrait pas rendre compte de l'expérience. Ce type de « transcendantalisme empirique », loin de supposer que l'analyse transcendantale réfute les résultats des connaissances positives, indique un rapport en principe plus « harmonieux » entre les deux.

Certes, il y a selon Foucault un problème avec ce rapport entre l'empirique et le transcendantal, mais il ne s'agit pas d'une contradiction : le problème semble plutôt être celui d'une perte de sens de la notion même d'une fondation transcendantale. Si les conditions de l'expérience peuvent être trouvées dans l'expérience elle-même, cela veut dire que la recherche transcendantale s'appuie sur une valeur épistémique déjà reconnue à l'expérience, et donc il serait faux de dire qu'il y a une « fondation » de la connaissance empirique par celle de type transcendantal ${ }^{2}$. Mais, comme on peut le noter, cette perte de spécificité de l'analyse transcendantale n'a rien à voir avec le problème mis au jour par Merleau-Ponty.

En bref, nous avons :

«La connaissance scientifique [des] facteurs empiriques qui nous déterminent profondément met en cause l'autonomie et l'intégrité absolue de la pensée que des philosophes préalables avaient proclamée. [...] Notre pensée est si profondément influencée par nos conditions économiques, biologiques et linguistiques qu'il devient difficile de voir de quelle façon elle est vraiment nôtre. [...] Foucault trace les diverses tentatives pour concilier les aspects incompatibles de constituant et constitué, déterminant et déterminé [...] 》 (L. Braver, A thing of this World. A History of Continental Anti-Realism, Evanston, Illinois, Northwestern University Press, 2007, p. 367).

${ }^{1}$ Les mots..., p. 261. Nous soulignons.

${ }^{2}$ Comme dit Béatrice Han, « comment l'a priori pourrait-il conserver un quelconque pouvoir fondateur, si une connaissance empirique suffit à le déterminer en retour? ». C'est une erreur de chercher " dans l'empirique une connaissance qui pourrait avoir valeur transcendantale, puisque la "flexion vers l'empirique" opérée suffit en ellemême à vider de tout son sens le concept de fondation transcendantale » (L'ontologie manquée de Michel Foucault, Grenoble, Jérôme Millon, 1998, p. 63). 
(a) l'analyse merleau-pontienne d'une incompatibilité dans deux aspects de la condition de l'homme, celui du sujet autonome et celui de l'objet conditionné ;

(b) l'analyse foucaldienne d'un rabaissement de la dimension de sujet transcendantal dans l'homme, en vertu de la contamination de la réflexion critique par des contenus empiriques.

En d'autres termes, il n'y a, dans Les mots et les choses, aucune référence de Foucault au point de départ merleau-pontien selon lequel l'homme, pris comme un sujet autonome et non un objet conditionné, serait la condition préalable de toute connaissance; ce n'est pas de cela qu'il s'agit lorsque l'archéologue parle de «transcendantalisme». Mais il y a plus : non seulement Foucault ne se prononce pas en faveur de la thèse de l'autonomie de la conscience comme condition de possibilité de la connaissance, mais, comme c'est connu, il prend précisément position contre ce postulat épistémologique, ce qui veut dire que ce qui apparaît comme un «dilemme» pour le phénoménologue ne l'est pas pour Foucault. Voyons les textes pertinents.

\section{Le sujet connaissant doit-il être autonome ? Sur une différence de principe entre Merleau-Ponty et Foucault}

Nous avons proposé plus haut une reconstruction de l'argument qui sert de point de départ au cours de Merleau-Ponty, et qui essaie de montrer pourquoi une approche « objectiviste » de la conscience humaine est insuffisante. On pourrait penser qu'une possible issue, plus ou moins évidente, à la conclusion sceptique que tire le phénoménologue serait de dire - contre ce que nous avons distingué comme la prémisse (4) - que la " portée » de la thèse de la dépendance de la pensée n'a pas besoin d'être aussi universelle que le croit le phénoménologue ; le savant peut en principe donner des bonnes raisons (et non pas des hypothèses ad hoc, du type que Max Weber reprochait au marxisme) pour expliquer pourquoi l'idée d'une conscience passive, qui n'a pas accès à ses propres fondements épistémiques, n'est pas nécessairement autoréférentielle; les thèses de l'ethnologue ou du psychologue n'ont pas besoin de se référer à la conscience scientifique elle-même, qui est d'un autre ordre que celle de l'expérience préscientifique. De cette manière, on pourrait admettre la prémisse (2) - selon laquelle une conscience passive n'est pas capable de connaissance - sans que cela implique la conséquence sceptique (5). Donc, précisément parce que cette solution est en principe possible, il est intéressant de noter comment Foucault la rejette explicitement, en insistant 
que la conscience en général doit être considérée comme passive ou conditionnée par rapport à l'histoire.

En effet, il serait peut-être moins polémique que la prémisse (4) d'admettre, d'abord, que « [q]uand on analyse le langage de l'homme, [...] on découvre des structures inconscientes qui gouvernent sans que nous le remarquions ou le voulions ", ou que, de la même manière, "[q]uand un psychanalyste analyse le comportement ou la conscience chez un individu », ce qu'il rencontre est «quelque chose comme une pulsion, un instinct, une impulsion ». Mais ce que Foucault veut faire, c'est appliquer les mêmes principes à l'étude de notre connaissance. C'est précisément ce qui, selon lui, le distingue de ceux qui n'acceptent pas sa démarche théorique :

Ce que j'ai voulu faire - et c'est peut-être cela qui a provoqué tant de protestations -, c'est montrer que dans l'histoire même du savoir humain on pouvait retrouver le même phénomène : l'histoire du savoir humain n'est pas restée entre les mains de l'homme. Ce n'est pas l'homme lui-même qui a consciemment créé l'histoire de son savoir, mais l'histoire du savoir et de la science humaine obéit elle-même à des conditions déterminantes qui nous échappent. Et, en ce sens, l'homme ne détient plus rien, ni son langage, ni sa conscience, ni même son savoir ${ }^{1}$.

Donc, Foucault adhère clairement à une forme de ce que Merleau-Ponty appellerait «historicisme» ou «psychologisme»: notre connaissance est déterminée par des mécanismes qui nous ne contrôlons pas, et c'est explicitement applicable au cas du savant lui-même. Cette application explicite réapparaît dans la "Réponse à une question ». Voici ce que Foucault dit sur ses critiques :

Ils ont eu sans doute assez de mal à reconnaître que leur histoire, leur économie, leurs pratiques sociales, la langue qu'ils parlent, la mythologie de leurs ancêtres, les fables mêmes qu'on leur racontait dans leur enfance obéissent à des règles qui ne sont pas toutes données à leur conscience; ils ne souhaitent guère qu'on les dépossède, en outre et par surcroît, de ce discours où ils veulent pouvoir dire immédiatement, sans distance, ce qu'ils pensent [...]; ils préféreront nier que le discours soit une pratique complexe et différenciée, obéissant à des règles et à transformations analysables $[\ldots]^{2}$.

${ }_{1}$ «En intervju med Michel Foucault», Dits et écrits, II, Paris, Gallimard, 2001, p. 687.

${ }_{2}^{2}$ « Réponse à une question », Dits et écrits, II, p. 725. 
Il est donc clair, d'un côté, que Foucault ne se soucie pas de revendiquer la possibilité de la connaissance en préservant, au moins dans une mesure limitée, l'autonomie de la conscience. Mais, d'un autre côté, Foucault a-t-il besoin de faire quelque chose de ce genre ? Il semble que non, précisément parce qu'il n'a pas besoin d'admettre la prémisse (2) de Merleau-Ponty, d'admettre qu'une conscience conditionnée est, par le fait même, aveugle, incapable de distinguer le vrai du faux, et que par conséquent la thèse du conditionnement de la conscience est de type sceptique. Ce qui importe ici et qui est caché par une interprétation comme celle de Sabot, lorsqu'il assimile les problèmes épistémologiques de Foucault à ceux de MerleauPonty -, c'est que l'archéologue peut définir autrement la distinction entre valeurs de vérité, et insister que cette distinction vaut encore comme telle, qu'elle ne disparaît pas, si on la conçoit comme une distinction interne à des règles historiques. Et c'est dans cette direction que Foucault se meut déjà dans $L$ 'ordre $d u$ discours, où il déclare que le partage entre le vrai et le faux - un partage, non pas une indistinction - doit être compris comme le résultat de l'application de critères historiquement contingents et variables ${ }^{1}$. Cette approche, c'est peut-être Martin Kusch qui l'a défendue de la manière la plus convaincante, en disant que le fait que les règles d'un jeu quelconque, disons les règles du jeu d'échecs, règlent les déplacements "corrects » et « incorrects » seulement pour ce jeu - et non pas pour d'autres - ne veut pas dire que, à l'intérieur du jeu d'échecs, cette distinction ne soit pas applicable. De même, on n'a pas besoin de dire que les règles selon lesquelles, dans une période historique donnée, on distingue le «vrai » du « faux » perdent cette capacité de discrimination si l'on ne les considère pas comme des règles universellement valables ${ }^{2}$.

Bien sûr, il ne va pas de soi que la notion de « vérité relative (à des règles) » soit une solution acceptable face aux «dangers » du scepticisme; ceux qui la critiquent peuvent encore répliquer que cette «solution» ne fait que déplacer la discussion vers le problème de savoir pourquoi on devrait accepter les règles elles-mêmes, et non pas d'autres, desquelles on tirerait, à

\footnotetext{
${ }^{1}$ Foucault, Michel, L'ordre du discours, Paris, Gallimard, 1971, p. 15ss.

${ }^{2}$ Cf. M. Kusch, Foucault's Strata and Fields, Dordrecht, Springer, 1991, p. 201. De la même manière, d'autres interprètes ont considéré que la vérité pouvait être définie à partir de Foucault comme quelque chose sanctionné par des règles dont l'application serait locale : en fait, même si Prado ne mentionne pas Kusch, sa définition de ce qui serait une " vérité relative » chez Foucault appelle à la même comparaison avec le jeu d'échecs. $C f$. C.G. Prado, Starting with Foucault. An Introduction to Genealogy, Westview Press, 2008, p. 121.
} 
son tour, d'autres " vérités » ${ }^{1}$. La question se pose, donc, de savoir si ces règles sont acceptées en raison de quelque chose comme une métarègle, ou si au contraire il nous est impossible de décider de réguler nos pratiques de connaissance selon certaines règles, qui nous sont simplement imposées dans leur facticité. Nous n'essayerons pas de répondre à ces questions ici. Mais nous croyons avoir montré que c'est dans cette direction que le débat entre Foucault et Merleau-Ponty peut être analysé de façon productive, sans occulter les différences épistémologiques cruciales entre les deux philosophes.

${ }^{1} C f$., par exemple, la discussion sur les «systèmes d'évidence » alternatifs dans H. Siegel, Relativism Refuted, Dordrecht, Springer, 1987, p. 29-30.

17 